\title{
Sparse Channel Estimation for MIMO-OFDM Two-Way Relay Network with Compressed Sensing
}

\author{
Aihua Zhang, ${ }^{1,2}$ Shouyi Yang, ${ }^{1}$ and Guan Gui ${ }^{3}$ \\ ${ }^{1}$ School of Information Engineering, Zhengzhou University, Zhengzhou 450001, China \\ ${ }^{2}$ School of Electronic and Information Engineering, Zhongyuan University of Technology, Zhengzhou 450007, China \\ ${ }^{3}$ Department of Communication Engineering, Graduate School of Engineering, Tohoku University, Sendai 980-8579, Japan
}

Correspondence should be addressed to Aihua Zhang; zhah00@yahoo.com.cn

Received 30 September 2012; Revised 7 February 2013; Accepted 12 February 2013

Academic Editor: Antonio Faraone

Copyright (C) 2013 Aihua Zhang et al. This is an open access article distributed under the Creative Commons Attribution License, which permits unrestricted use, distribution, and reproduction in any medium, provided the original work is properly cited.

\begin{abstract}
Accurate channel impulse response (CIR) is required for equalization and can help improve communication service quality in nextgeneration wireless communication systems. An example of an advanced system is amplify-and-forward multiple-input multipleoutput two-way relay network, which is modulated by orthogonal frequency-division multiplexing. Linear channel estimation methods, for example, least squares and expectation conditional maximization, have been proposed previously for the system. However, these methods do not take advantage of channel sparsity, and they decrease estimation performance. We propose a sparse channel estimation scheme, which is different from linear methods, at end users under the relay channel to enable us to exploit sparsity. First, we formulate the sparse channel estimation problem as a compressed sensing problem by using sparse decomposition theory. Second, the CIR is reconstructed by CoSaMP and OMP algorithms. Finally, computer simulations are conducted to confirm the superiority of the proposed methods over traditional linear channel estimation methods.
\end{abstract}

\section{Introduction}

Two-way relay network (TWRN) has attracted great attention because it can improve spectral efficiency unlike a oneway relay network [1]. In a typical TWRN, two terminals, $\mathbb{T}_{1}$ and $\mathbb{T}_{2}$, exchange information through the assistance of an intermediate relay $\mathbb{R}$ via amplify-and-forward $(\mathrm{AF})$. The TWRN requires two time slots to exchange their information. At the first time slot, both terminals send their data to the relay simultaneously, and then $\mathbb{R}$ amplifies the superimposed signal and broadcasts it to the end terminals during the second time slot. After receiving the signal from $\mathbb{R}$, the end nodes remove their own interference and perform a coherent detection process to recover the data transmitted from the other node. Compared with the basic single-antenna relay channel, the multiple-input multiple-output (MIMO) relay channel provides the high capacity of MIMO communication with the coverage extension capability of relay nodes. MIMO relay channel is one of the most promising solutions as it increases channel capacity and network reliability and effectively combats multipath fading. Furthermore, the orthogonal frequency-division multiplexing (OFDM) modulation technique provides efficient bandwidth utilization and robustness against time-dispersive channels. Therefore, the MIMO-OFDM-TWRN is a promising technique for nextgeneration wireless communication systems.

The MIMO-OFDM-TWRN system faces more challenges because channel estimation is required not only for data detection but also for self-data cancellation at the two terminals. Linear channel estimation methods have been proposed for MIMO-OFDM-TWRN. In [2], channel estimation by using least squares (LS) was proposed, and the blockbased training design method was considered to achieve the optimal linear channel estimator. In addition, channel information estimation which uses the expectation conditional maximization (ECM) algorithm was proposed in [3]. However, both proposed methods do not take advantage of channel sparsity, and they result in performance loss of MIMO-OFDM-TWRN. In the development of channel modeling, numerous measurements demonstrated that multipath wireless channels tended to exhibit a cluster or sparse structure in which majority of the channel taps end up 


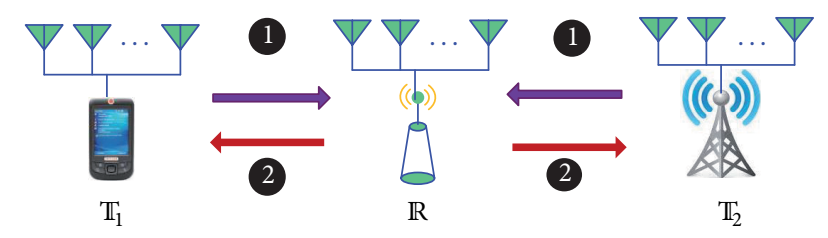

FIGURE 1: MIMO-OFDM-TWRN: (1) multiaccess stage, (2) broadcasting stage.

being either zero or below the noise floor, especially when operating at large bandwidths and signaling durations and/or with numbers of antenna elements [4-6]. In addition, with the theoretical development of compressed sensing (CS) [7], numerous researchers have proposed sparse channel estimation methods for point-to-point (P2P) communication systems, including single-antenna [8] or multiple-antenna [9] systems. Sparse channel estimation methods [10, 11] on cooperative networks have also been investigated. All channel estimation methods are limited to either singleantenna or multiple-antenna systems for traditional P2P transmission and single-antenna relay channel. To the best of our knowledge, corresponding work has not been conducted for MIMO-OFDM-TWRN.

In this study, we focus on the TWRN with multiple antennas at relay $\mathbb{R}$ and both end nodes, which is modulated by OFDM. We introduce the MIMO-OFDM-TWRN channel model and formulate composite channels estimation as a compressed sensing problem by using sparse decomposition theory. Then, the composite channel is reconstructed by CoSaMP [12] and OMP [13] algorithms. Finally, we verify the proposed methods via computer simulations. This study contributes to the field by introducing a sparse channel estimation technique with compressed sensing for MIMOOFDM-TWRN and the use of the sparse structure information in the CIR by the end users.

The remainder of this paper is organized as follows. Section 2 describes the system model. Section 3 provides details on the proposed algorithm. The simulation results are reported in Section 4, and the conclusions are provided in Section 5 .

\section{System Model}

2.1. Relay Transmission Model. Figure 1 shows a typical TWRN with two source nodes, $\mathbb{T}_{1}$ and $\mathbb{T}_{2}$, and one relay node, $\mathbb{R}$. The two terminal users exchange information with the assistance of the relay node. $\mathbb{T}_{1}$ and $\mathbb{T}_{2}$ each have $N_{T}$ antennas, and $\mathbb{R}$ has $N_{R}$ antennas. The bidirectional communication is performed in two phases, as shown in Figure 1. In phase $I, \mathbb{T}_{1}$ and $\mathbb{T}_{2}$ send their signal simultaneously to the relay node $\mathbb{R}$. Then, the relay amplifies the received signals and broadcasts them to the source nodes $\mathbb{T}_{1}$ and $\mathbb{T}_{2}$ in phase II.

The channel between the $m$ th antenna of $\mathbb{T}_{i}$ and the $r$ th antenna of $\mathbb{R}$ is assumed to be a quasi-static frequencyselective fading channel with an impulse response denoted by $\mathbf{h}_{r, m}^{i}=\left[h_{r, m}^{i}(0), h_{r, m}^{i}(1), \ldots, h_{r, m}^{i}(L-1)\right]^{T} \in C^{L \times 1}$, where $i=1,2$ and $L$ is the channel length. All channels $\mathbf{h}_{r, m}^{i},\left(i=1,2 ; m=1, \ldots, N_{T} ; r=1, \ldots, N_{R}\right)$ are assumed as zero-mean circularly symmetric complex Gaussian random variables with variance $\left(\sigma_{r, m}^{i}\right)^{2}$ and are independent of each other. The average transmission powers of $\mathbb{T}_{1}, \mathbb{T}_{2}$, and $\mathbb{R}$ are $P_{1}, P_{2}$, and $P_{R}$, respectively. For the time being, we assume perfect synchronization among three terminals.

2.2. Received Signals at the Relay Node and the End Users. The training signal vectors transmitted from the $m$ th antenna of $\mathbb{T}_{1}$ and $\mathbb{T}_{2}$ are denoted by $s_{m}^{i}$. We assume that $E\left\{\left\|s_{m}^{i}\right\|^{2}\right\}=$ $P_{i}$. In phase I, the signal vectors $\mathbf{s}_{m}^{1}$ and $\mathbf{s}_{m}^{2}$ are processed by an inverse fast Fourier transform as $\widetilde{\mathbf{s}}_{m}^{i}=\mathbf{F}^{H} \mathbf{s}_{m}^{i}=$ $\left[\vec{s}_{m}^{i}(0), \vec{s}_{m}^{i}(1), \ldots, \vec{s}_{m}^{i}(N-1)\right]$, where $F$ is the discrete Fourier transformation (DFT) matrix with the $(m, n)$ th entity given by $\mathbf{F}=1 / \sqrt{N} e^{-j 2 \pi m n / N},(m, n=0,1, \ldots, N)$. To avoid interblock interference, $\widetilde{\mathbf{s}}_{m}^{i}$ is the cyclic prefix (CP) of length $L_{p}$ added before being transmitted, and $L_{p}$ should satisfy $L_{p} \geq$ $L-1$. The received signal vector at the node $\mathbb{R}$ after removing $\mathrm{CP}$ can be written as

$$
\mathbf{y}_{R}=\mathbf{H}^{1} \widetilde{\boldsymbol{s}}^{1}+\mathbf{H}^{2} \widetilde{\boldsymbol{s}}^{2}+\mathbf{w}
$$

where

$$
\begin{gathered}
\tilde{\mathbf{s}}^{1}=\left[\left(\tilde{s}_{1}^{1}\right)^{T},\left(\tilde{s}_{2}^{1}\right)^{T}, \ldots\left(\tilde{s}_{N_{T}}^{1}\right)^{T}\right]^{T}, \\
\widetilde{\mathbf{s}}^{2}=\left[\left(\tilde{s}_{1}^{2}\right)^{T},\left(\tilde{s}_{2}^{2}\right)^{T}, \ldots\left(\tilde{s}_{N_{T}}^{2}\right)^{T}\right]^{T}, \\
\mathbf{H}^{1}=\left[\begin{array}{cccc}
\mathbf{H}_{1,1}^{1} & \mathbf{H}_{1,2}^{1} & \cdots & \mathbf{H}_{1, N_{T}}^{1} \\
\mathbf{H}_{2,1}^{1} & \mathbf{H}_{2,2}^{1} & \cdots & \mathbf{H}_{2, N_{T}}^{1} \\
\vdots & \vdots & \vdots & \vdots \\
\mathbf{H}_{N_{R}, 1}^{1} & \mathbf{H}_{N_{R}, 2}^{1} & \cdots & \mathbf{H}_{N_{R}, N_{T}}^{1}
\end{array}\right], \\
\mathbf{H}^{2}=\left[\begin{array}{cccc}
\mathbf{H}_{1,1}^{2} & \mathbf{H}_{1,2}^{2} & \cdots & \mathbf{H}_{1, N_{T}}^{2} \\
\mathbf{H}_{2,1}^{2} & \mathbf{H}_{2,2}^{2} & \cdots & \mathbf{H}_{2, N_{T}}^{2} \\
\vdots & \vdots & \vdots & \vdots \\
\mathbf{H}_{N_{R}, 1}^{2} & \mathbf{H}_{N_{R}, 2}^{2} & \cdots & \mathbf{H}_{N_{R}, N_{T}}^{2}
\end{array}\right], \\
\mathbf{w}=\left[\begin{array}{llll}
\mathbf{w}_{1}^{T} \mathbf{w}_{2}^{T} & \cdots & \mathbf{w}_{N_{R}}^{T}
\end{array}\right]^{T} .
\end{gathered}
$$

The matrix $\mathbf{H}_{r, m}^{i}$ is an $N \times N$ circulant matrix in which the first column takes the form $\mathbf{h}_{r, m}^{i}=\left[h_{r, m}^{i}(0) h_{r, m}^{i}(1) \cdots h_{r, m}^{i}(L-\right.$ 1) $\left.0_{1 \times(N-L)}\right]^{T}$. The noise vector $\mathbf{w}$ is a complex Gaussian random vector with zero mean and covariance matrix $E\left\{\mathbf{w w}^{H}\right\}=$ $N_{0} \mathbf{I}_{N}$.

In phase II, the vector $y_{R}$ is amplified by a real factor $\beta$, and $\beta^{2} E\left\{\mathbf{y}^{H} \mathbf{y}\right\}=N_{R} \times P_{R}$. The factor $\beta$ is given by

$$
\beta=\sqrt{\frac{P_{R}}{\sum_{r=1}^{N_{R}} \sum_{m=1}^{N_{T}} \sum_{l=0}^{L-1}\left[\sigma_{r, m}^{1}(l)+\sigma_{r, m}^{2}(l)\right]+N_{0}}},
$$

where $\sigma_{r, m}^{i}(l)=E\left[\left|\mathbf{h}_{r, m}^{i}(l)\right|^{2}\right], i=1,2$. 
The vector $\beta \mathbf{y}_{R}$ is CP-added before being transmitted back to $\mathbb{T}_{1}$ and $\mathbb{T}_{2}$. Only the channel estimation problem at $\mathbb{T}_{1}$ is considered in this study. A similar procedure can be applied at $\mathbb{T}_{2}$. The received signal at $\mathbb{T}_{1}$, which is from relay $\mathbb{R}$ after removing $\mathrm{CP}$, can be written as [3]

$$
\mathbf{y}_{T_{1}}=\beta \mathbf{G}\left(\mathbf{H}^{1} \widetilde{\boldsymbol{s}}^{1}+\mathbf{H}^{2} \widetilde{\boldsymbol{s}}^{2}\right)+\mathbf{n}_{T_{1}},
$$

where

$$
\mathbf{G}=\left[\begin{array}{cccc}
\mathbf{H}_{1,1}^{1} & \mathbf{H}_{2,1}^{1} & \cdots & \mathbf{H}_{N_{R}, 1}^{1} \\
\mathbf{H}_{1,2}^{1} & \mathbf{H}_{2,2}^{1} & \cdots & \mathbf{H}_{N_{R}, 2}^{1} \\
\vdots & \vdots & \vdots & \vdots \\
\mathbf{H}_{1, N_{T}}^{1} & \mathbf{H}_{2, N_{T}}^{1} & \cdots & \mathbf{H}_{N_{R}, N_{T}}^{1}
\end{array}\right]
$$

and $\mathbf{n}_{T_{1}}=\beta \mathbf{G w}+\mathbf{n}, \mathbf{n}=\left[n_{1}^{T} n_{2}^{T} \cdots n_{N_{R}}^{T}\right]^{T}$.

According to the matrix theory, matrices $\mathbf{H}_{r, m}^{i}$ can be decomposed as $\mathbf{H}_{r, m}^{i}=\mathbf{F}^{H} \Lambda_{r, m}^{i} \mathbf{F}$ [14], where $\Lambda_{r, m}^{i}=$ $\operatorname{diag}\left\{\mathbf{H}_{r, m}^{i}(0), \mathbf{H}_{r, m}^{i}(1), \ldots, \mathbf{H}_{r, m}^{i}(N-1)\right\}$ and $\mathbf{H}_{r, m}^{i}(c)=$ $\sum_{l=0}^{L-1} \mathbf{h}_{r, m}^{i} e^{-j(2 \pi l c / N)},(c=0,1, \ldots, N-1)$

$$
\begin{aligned}
& \beta \mathbf{H}_{r_{1}, m_{1}}^{1} \mathbf{H}_{r_{2}, m_{2}}^{1}=\mathbf{F}^{H} \beta \boldsymbol{\Lambda}_{r_{1}, m_{1}}^{1} \Lambda_{r_{2}, m_{2}}^{1} \mathbf{F}, \\
& \beta \mathbf{H}_{r_{1}, m_{1}}^{1} \mathbf{H}_{r_{2}, m_{2}}^{2}=\mathbf{F}^{H} \beta \boldsymbol{\Lambda}_{r_{1}, m_{1}}^{1} \Lambda_{r_{2}, m_{2}}^{2} \mathbf{F},
\end{aligned}
$$

where $r_{1}$ and $r_{2}=1,2, \ldots, N_{R}$, and $m_{1}$ and $m_{2}=1,2, \ldots, N_{T}$. Equations (6) are two circulant matrices that have the first columns of $\left[\beta\left(\mathbf{h}_{r_{1} m_{1}}^{1} * \mathbf{h}_{r_{2} m_{2}}^{1}\right) 0_{1 \times(N-2 L+1)}\right]^{T}$ and $\left[\beta\left(\mathbf{h}_{r_{1} m_{1}}^{1} *\right.\right.$ $\left.\left.\mathbf{h}_{r_{2} m_{2}}^{2}\right) 0_{1 \times(N-2 L+1)}\right]^{T}$. Thus, by normalizing DFT of $\mathbf{y}_{T_{1}}$, system model (4) can be rewritten as

$$
\begin{aligned}
\mathbf{y}_{1} & =(\mathbf{I} \otimes \mathbf{F}) y_{T_{1}} \\
& =\sum_{r=1}^{N_{R}} \sum_{m=1}^{N_{T}} \beta \boldsymbol{\Lambda}_{r_{1}, m_{1}}^{1}\left(\Lambda_{r_{2}, m_{2}}^{1} s^{1}+\Lambda_{r_{2}, m_{2}}^{2} s^{2}\right)+\mathbf{F} n_{T_{1}} .
\end{aligned}
$$

From (7), we define composite channels $g$ and $q$, which are given, respectively, as

$$
\begin{gathered}
\mathbf{g}=\beta\left(\mathbf{h}_{r, m}^{1} \otimes \mathbf{h}_{r, n}^{1}\right), \\
\beta \boldsymbol{\Lambda}_{r_{1}, m_{1}}^{1} \boldsymbol{\Lambda}_{r_{2}, m_{2}}^{1}=\operatorname{diag}(\mathbf{W g}), \\
\mathbf{q}=\beta\left(\mathbf{h}_{r, m}^{1} \otimes \mathbf{h}_{r, n}^{2}\right), \\
\beta \boldsymbol{\Lambda}_{r_{1}, m_{1}}^{1} \boldsymbol{\Lambda}_{r_{2}, m_{2}}^{2}=\operatorname{diag}(\mathbf{W q}) .
\end{gathered}
$$

Then, (7) can be expressed as

$$
\mathbf{y}_{1}=\mathbf{S k}+\mathbf{F} \mathbf{n}_{T_{1}},
$$

where $\mathbf{S}=\left[\mathbf{s}^{1} \mathbf{s}^{2}\right], \mathbf{k}=\left[\mathbf{g}^{T} \mathbf{q}^{T}\right]$, and $\mathbf{W}$ is a matrix which takes the first $(2 L-1)$ columns of $\sqrt{N} \mathbf{F}$.

\section{Sparse Channel Estimation}

3.1. Overview of Compressed Sensing. Compressed sensing (CS) describes a new signal acquisition theory in which sparse high dimensional vectors can be accurately recovered from a small number of linear observations. CS has been applied in various areas, such as imaging, radar, speech recognition, and data acquisition. In communications, an immediate application of CS is wireless sparse multipath channel estimation. Detailed descriptions can be found in [7].

In this paper, we consider the linear model as (9). According to the CS, if an unknown signal vector satisfies the sparse or approximate sparse requirements, the conditions under which CS succeeds depends on the structure of the measurement matrix $\mathbf{S}$. Thus, these kinds of unknown signals can be robustly reconstructed from observation signal $\mathbf{y}_{1}$. However, the sparsest solution is always a nondeterministic polynomial-time hard (NP-hard) problem. According to recent theoretical results, the observation signal can be used to efficiently recover any "sparse enough" signal provided that the matrix $\mathbf{S}$ satisfies the so-called restricted isometric property (RIP) $[15,16]$. We suppose that $S$ is a $n \times p$ complexvalued measurement matrix that has unit $\ell_{2}$-norm columns. The $\mathbf{S}$ satisfies the RIP of order $d$ with parameter $\delta_{d} \in(0,1)$, which can satisfy the inequality

$$
\left(1-\delta_{d}\right)\|\mathbf{k}\|_{2}^{2} \leq\|\mathbf{S k}\|^{2} \leq\left(1+\delta_{d}\right)\|\mathbf{k}\|_{2}^{2},
$$

where $\|\mathbf{k}\|_{2}^{2}$ denotes the $\ell_{2}$-norm, which is given by $\|\mathbf{k}\|_{2}^{2}=$ $\sum\left|\mathbf{h}_{r, m}^{i}\right|^{2}$. If (10) is satisfied, the training sequence is said to satisfy the RIP of order $d$, and the accurate channel estimator with high probability can be obtained by using CS methods. Although verifying whether a given matrix satisfies this condition is difficult, many matrices satisfy the restricted isometry constant (RIC) with high property and few measurements. In particular, it has been shown exponentially with high probability that the random Gaussian, Bernoulli, and partial Fourier matrices satisfy the RIC with a number of measurements that are nearly linear in the sparsity level.

3.2. Compressed Channel Estimation (CCE). Since the channel impulse responses $\mathbf{h}_{r, m}^{i}$ are sparse enough, their cooperation convoluted channel $g$ and $q$ have been verified to be sparse or approximate sparse [17]. In this case, we use two greedy algorithms, that is, orthogonal matching pursuit (OMP) [13] and compressive sampling matching pursuit (CoSaMP) [12], which select each dominant coefficient in channel through iteration. We also present the LS channel estimator for comparison. The LS estimator (known position) is given by numerous practical algorithms for channel estimation. The LS estimator is expressed as

$$
\widehat{\mathbf{k}}= \begin{cases}\mathbf{S}_{T}^{\dagger} \mathbf{y}_{1}, & T \subseteq \operatorname{supp}(\mathbf{k}), \\ 0, & \text { others },\end{cases}
$$

where $\operatorname{supp}(\mathbf{k})$ denotes the nonzero taps that support the channel vector $\mathbf{k}, \mathbf{S}_{T}$ is the submatrix constructed from the columns of $\mathbf{S}$, and $T$ denotes the selected subcolumns which correspond to the nonzero index set of the convoluted 
channel vector $h$. The mean square error (MSE) of the LS estimator $\widehat{\mathbf{k}}$ is given by

$$
\widehat{\mathbf{k}} \triangleq \mathbf{S}^{\dagger} \mathbf{y}_{1}=\mathbf{k}+\mathbf{S}^{\dagger} \mathbf{F} \mathbf{n}_{T}
$$

where $\mathbf{S}^{\dagger}$ is the pseudo-inverse of $\mathbf{S}$ and is given by $\mathbf{S}^{\dagger}=$ $\left(\mathbf{S}^{H} \mathbf{S}\right)^{-1} \mathbf{S}^{H}$. The MSE of the LS channel estimate is given by MSE $=E\left\{\|\widehat{\mathbf{k}}-\mathbf{k}\|^{2}\right\}=E\left\{\left\|\mathbf{S}^{\dagger} \mathbf{F} \mathbf{n}_{T}\right\|^{2}\right\}$, which can also be written as

$$
\operatorname{MSE}(\widehat{\mathbf{k}})=\sigma_{n}^{2} \operatorname{tr}\left\{\left(\mathbf{S}^{\dagger} \mathbf{S}\right)^{-1}\right\} .
$$

By utilizing CS recovery algorithms for compressive channel estimation, we propose CCE-OMP and CCECoSaMP. The two estimation methods for convoluted channels are described as follows.

3.2.1. CoSaMP Estimator $\widehat{\mathbf{k}}_{\text {CoSaMP. Given }} y_{1}, F$ and $W$, and training signal matrix $\mathbf{S}=\operatorname{diag}\left(\mathbf{F} \mathbf{s}^{i}\right) \mathbf{W}$, the maximum number of dominant channel coefficients is assumed as $d$. The CCE-CoSaMP is performed as follows.

Initialization. We set the nonzero coefficient index as $T_{0}=$ $\emptyset$, the residual estimation error $\mathbf{r}_{0}=\mathbf{y}_{1}$, and the initialize iteration counter as $l=1$.

Identification. We select a column index $n_{l}$ of $\mathbf{S}$ that is most correlated with the residual

$$
n_{l}=\left|\left\langle r_{l-1}, \mathbf{S}_{n}\right\rangle\right|, \quad T_{l}=T_{l-1} \cup n_{l} .
$$

We use the LS method to calculate a channel estimator as $\mathbf{T}_{\mathrm{LS}}=\arg \min \left\|\mathbf{y}_{1}-\mathbf{S k}\right\|$ and select a maximum of $T$ dominant taps denoted by $\mathbf{k}_{\mathrm{LS}}$. The positions of the selected dominant taps in this substep are denoted by $T_{\mathrm{LS}}$.

Merge. The positions of the dominant taps are merged by $T_{l}=$ $T_{\mathrm{LS}} \cup T_{l}$.

Estimation. We compute the best coefficient for approximating the channel vector with chosen columns

$$
\mathbf{k}_{l}=\arg \min _{k}\left\|\mathbf{y}-\mathbf{S}_{T_{l}} \mathbf{k}\right\|_{2} .
$$

Pruning. We select the $T_{l}$ largest channel coefficients

$$
k_{l}=[k]_{d}
$$

and replace the left taps $T \backslash T_{l}$ by zero.

Iteration. We update the estimation error

$$
\mathbf{r}_{l}=\mathbf{y}-\widetilde{\mathbf{X}}_{T_{l}} \mathbf{k}_{l} .
$$

We increase the iteration counter $k$. We repeat (14) to (17) until the stopping criterion holds, and then set $\widehat{\mathbf{k}}_{\mathrm{CoSaMP}}=\mathbf{k}_{l}$.

3.2.2. OMP Estimator $\widehat{\mathbf{k}}_{\mathrm{OMP}}$. Given the received signal $\mathbf{y}_{\mathbf{1}}, \mathbf{W}$, $\mathbf{F}$, and $\mathbf{s}^{i}$, the CCE-OMP estimator runs as follows.
Initialize. We set the nonzero coefficient index as $T_{0}=\emptyset$, the residual estimation error $\mathbf{r}_{0}=\mathbf{y}_{1}$, and the initialize iteration counter as $l=1$.

Identification. We select a column index $n_{l}$ of $\mathbf{s}^{i}$ that is most correlated with the residual

$$
\begin{aligned}
n_{l} & =\left|\mathbf{r}_{l-1}, \mathbf{S}_{n}\right|, \\
T_{l} & =\mathrm{T}_{l-1} \cup n_{l}, \\
\mathbf{k}_{l} & =\arg \min _{k}\left|\mathbf{y}_{1}-\mathbf{S}_{T_{l}}\right| .
\end{aligned}
$$

Estimation. We compute the best coefficient for approximating the channel vector with chosen columns

$$
\mathbf{k}_{l}=\arg \min _{k}\left\|\mathbf{y}_{1}-\mathbf{S}_{T_{l}} \mathbf{k}\right\|_{2} .
$$

Iteration. We update the estimation error

$$
\mathbf{r}_{l}=\mathbf{y}_{1}-\mathbf{S}_{T_{l}} \mathbf{k}_{l} .
$$

We increase the iteration counter $l$. We repeat (18) to (20) until the stopping criterion holds, and then set $\widehat{\mathbf{k}}_{\mathrm{OMP}}=\mathbf{k}_{l}$.

\section{Simulation Results}

In this section, we present the simulation results and analyze the performance of compressive channel estimation in a MIMO-OFDM two-way relay network. We compare the performance of the proposed estimators with that of an LSbased linear estimator and adopt 10,000 independent Monte Carlo runs for averaging. We consider the MIMO relay network with $N_{\mathrm{T}}=N_{R}=2$ antennas; the number of carriers is 128 . All channels have the same length $(P=32)$, and the positions of the nonzero channel taps are randomly generated. QPSK modulation is used. Transmit power is set as $P_{1}=P_{2}=P$, and $\mathrm{AF}$ relay power is set as $P_{R}=P$. The signal-to-noise ratio is defined as $a$ as $10 \log \left(P / \sigma_{n}^{2}\right)$. When the number of nonzero taps in cooperation channels $\mathbf{h}_{r, m}^{i},(i=$ 1,$\left.2 ; r=1,2, \ldots, N_{R} ; m=1,2, \ldots, N_{T}\right)$ is changed, the simulation results are shown in Figures 2 to 5 .

The channel estimators are evaluated via the average MSE, which is defined by

$$
\text { average } \operatorname{MSE}(\Delta \mathbf{k})=\frac{\|\mathbf{k}-\widehat{\mathbf{k}}\|_{2}^{2}}{M(2 L-1)},
$$

where $\mathbf{k}$ and $\widehat{\mathbf{k}}$ denote the channel vector and its estimator, respectively, $M$ is the number of Monte Carlo runs, and $(2 L-$ 1 ) is the overall length of channel vector $k$. In Figure 2, the number of nonzero taps of $\mathbf{h}_{r, m}^{i},(i=1,2)$ is set to 2 , and the cooperation convoluted channel also has sparsity. Figure 2 shows that the performance of the proposed CCE methods is significantly better than that of the LS estimator and is close to the ideal LS estimator by using the known position of the channel. In Figure 3, the number of nonzero taps of $\mathbf{h}_{r, m}^{i},(i=1,2)$ is set as 4 . 


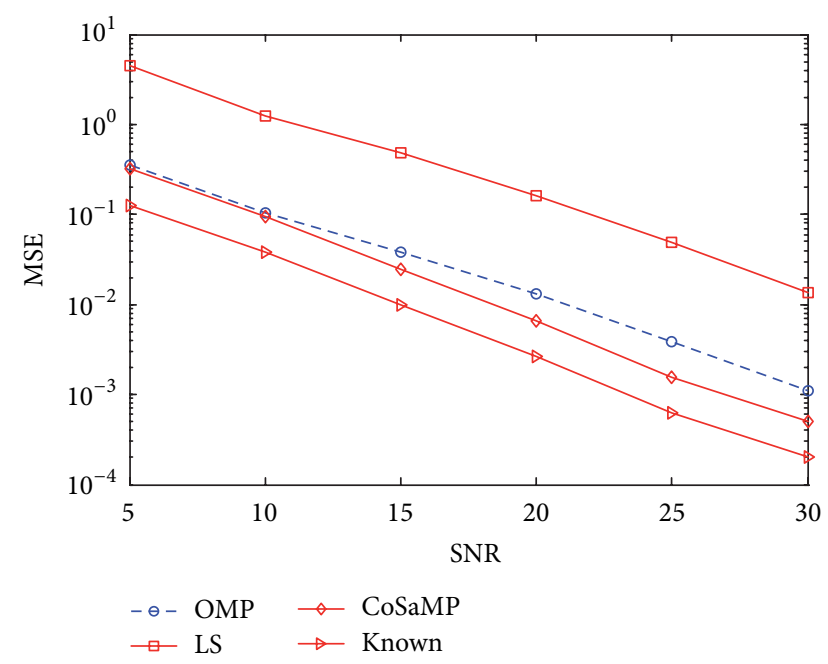

FIGURE 2: MSE performance of channel estimation at $\mathbb{T}_{1}$ for the case when $d=2$.

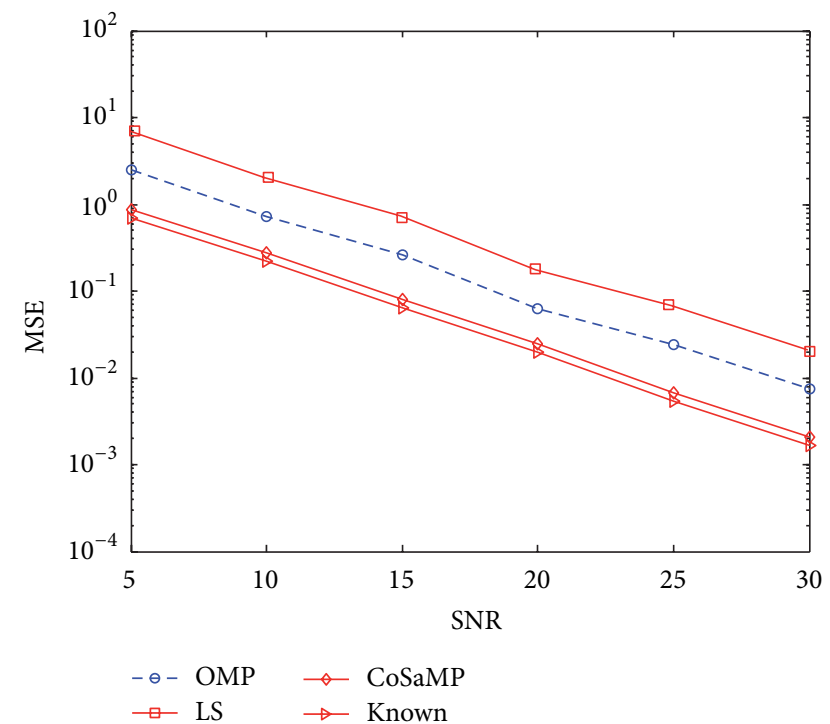

FIGURE 3: MSE performance of channel estimation at $\mathbb{T}_{1}$ when $d=4$.

A comparison between the simulation results in the two figures (Figures 2 and 3 ) shows that the proposed estimators can exploit the channel sparseness. Notably, if channels are dense rather than sparse, all proposed estimators will have the same performance as the LS estimator.

We also compare the performance of the proposed CCECoSaMP estimator with that of the ECM estimator algorithm in this section. Figures 4 and 5 show that when channel impulse responses $\mathbf{h}_{r, m}^{i}$ are sparse enough, the proposed CCE estimator performs significantly better than the ECM algorithm. However, as the number of nonzero taps of all the channels increases, the performance of CCE-CoSaMP is closer to that of the ECM algorithm.

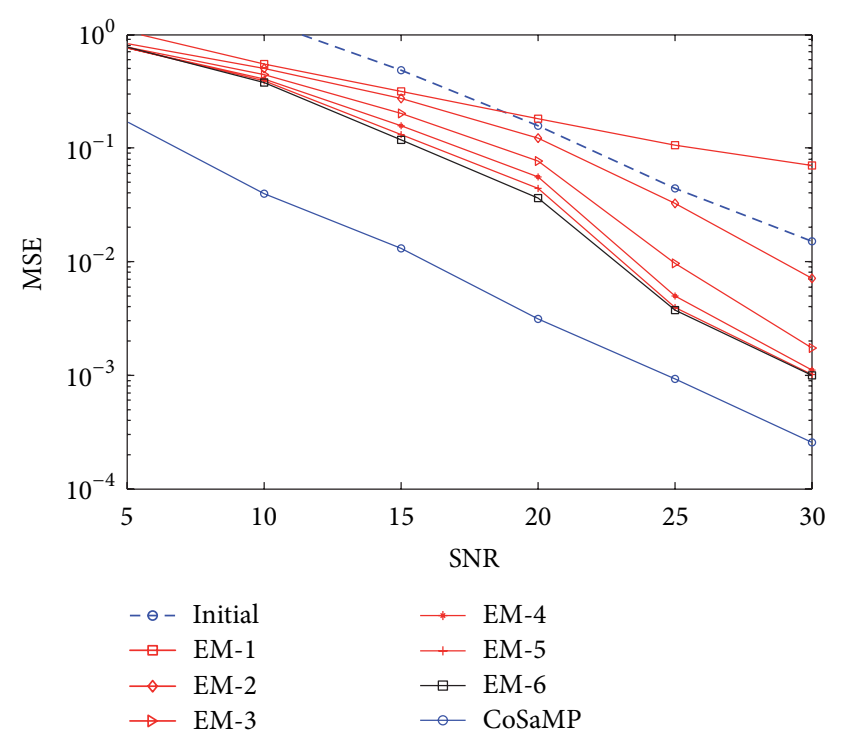

FIGURE 4: MSE performance of channel estimation at $\mathbb{T}_{1}$ for when channel impulse responses $\mathbf{h}_{r, m}^{i}$ are sparse enough $(d=2)$.

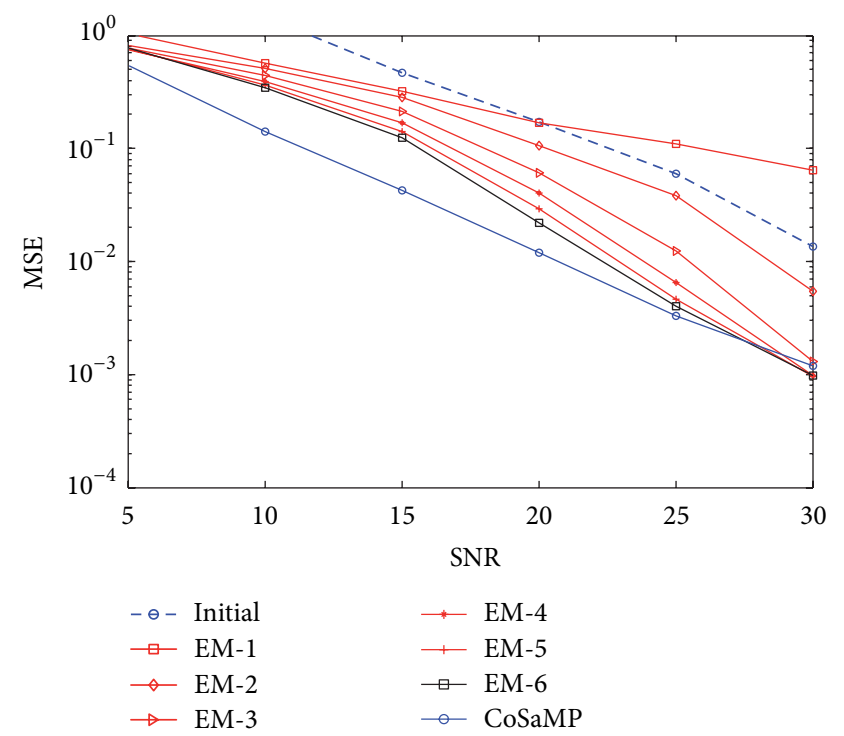

FIGURE 5: MSE performance of channel estimation at $\mathbb{T}_{1}$ for when channel impulse responses $\mathbf{h}_{r, m}^{i}$ are sparse $(d=4)$.

\section{Conclusion}

This paper investigated the channel estimation problem in sparse multipath MIMO two-way relay networks that adopt the OFDM technique. To address the shortcomings of conventional linear channel estimation methods, we proposed compressed channel estimation methods for MIMOOFDM two-way relay networks under the AF protocol. The sparseness of convoluted sparse channels was demonstrated by a measure function. The proposed methods exploited the sparsity in the MIMO TWRN channel. The simulation results confirmed the superior performance of the proposed method 
compared with conventional linear methods, for example, LS and ECM.

\section{Acknowledgment}

This study is funded by the National Natural Science Foundation of China (Grant nos. 61071175, 61202499, 61271421, and 61152004).

\section{References}

[1] P. Larsson, N. Johansson, and K. E. Sunell, "Coded bidirectional relaying," in Proceedings of the 63rd IEEE Vehicular Technology Conference (VTC '06), pp. 851-855, May 2006.

[2] Z. Fang and J. Shi, "Least square channel estimation for two-way relay MIMO-OFDM systems," ETRI Journal, vol. 33, no. 5, pp. 806-809, 2011.

[3] T. H. Pham, Y. C. Liang, H. K. Garg, and A. Nallanathan, "Joint channel estimation and data detection for MIMO-OFDM twoway relay networks," in Proceedings of the 53rd IEEE Global Communications Conference (GLOBECOM '10), Miami, Fla, USA, December 2010.

[4] W. U. Bajwa, A. Sayeed, and R. Nowak, "Sparse multipath channels: modeling and estimation," in Proceedings of the 13th IEEE Digital Signal Processing Workshop and 5th IEEE Signal Processing Education Workshop (DSP/SPE '09), pp. 320-325, Marco Island, Fla, USA, January 2009.

[5] N. Czink, X. Yin, H. Özcelik, M. Herdin, E. Bonek, and B. H. Fleury, "Cluster characteristics in a MIMO indoor propagation environment," IEEE Transactions on Wireless Communications, vol. 6, no. 4, pp. 1465-1474, 2007.

[6] Y. Zhou, M. Herdin, A. M. Sayeed, and E. Bonek, "Experimental study of MIMO channel statistics and capacity via the virtual channel representation," Tech. Rep., University of WisconsinMadison, February 2007.

[7] D. L. Donoho, "Compressed sensing," IEEE Transactions on Information Theory, vol. 52, no. 4, pp. 1289-1306, 2006.

[8] W. U. Bajwa, J. Haupt, G. Raz, and R. Nowak, "Compressed channel sensing," in Proceedings of the 42nd Annual Conference on Information Sciences and Systems (CISS '08), pp. 19-21, Princeton, NJ, USA, 2008.

[9] W. U. Bajwa, J. Haupt, A. M. Sayeed, and R. Nowak, "Compressed channel sensing: a new approach to estimating sparse multipath channels," Proceedings of the IEEE, vol. 98, no. 6, pp. 1058-1076, 2010.

[10] A. Zhang, G. Gui, and S. Yang, "Compressive channel estimation for OFDM cooperation networks," Research Journal of Applied Sciences, vol. 4, no. 8, pp. 897-901, 2012.

[11] G. Gui, Z. Chen, Q. Meng, Q. Wan, and F. Adachi, "Compressed channel estimation for sparse multipath two-way relay networks," International Journal of Physical Sciences, vol. 6, no. 12, pp. 2782-2788, 2011.

[12] D. Needell and J. A. Tropp, "CoSaMP: iterative signal recovery from incomplete and inaccurate samples," Communications of the ACM, vol. 53, no. 12, pp. 93-100, 2010.

[13] J. A. Tropp and A. C. Gilbert, "Signal recovery from random measurements via orthogonal matching pursuit," IEEE Transactions on Information Theory, vol. 53, no. 12, pp. 4655-4666, 2007.
[14] R. M. Gray, “Toeplitz and circulant matrices: a review," Foundations and Trends in Communications and Information Theory, vol. 2, no. 3, pp. 155-239, 2006.

[15] E. J. Candès, "The restricted isometry property and its implications for compressed sensing," Compte Rendus de l'Academie des Sciences, Paris, Series I, vol. 346, no. 9-10, pp. 589-592, 2008.

[16] E. J. Candes and T. Tao, "Decoding by linear programming," IEEE Transactions on Information Theory, vol. 51, no. 12, pp. 4203-4215, 2005.

[17] G. Gui, W. Peng, A. Mehbodniya, and F. Adachi, "Compressed channel estimation for sparse multipath non-orthogonal amplify-and-forward cooperative networks," in Proceedings of the 75th IEEE Vehicular Technology Conference (VTC '12), Yokohama, Japan, May 2012. 

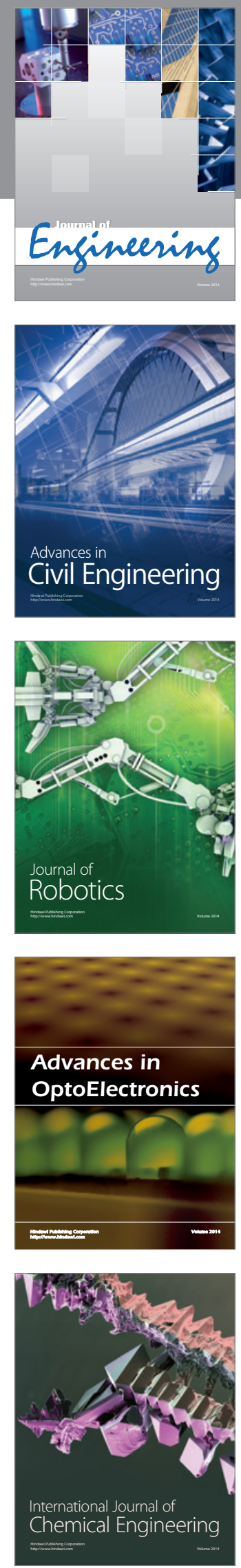

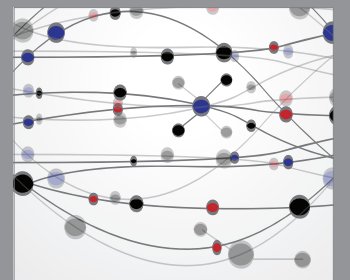

The Scientific World Journal
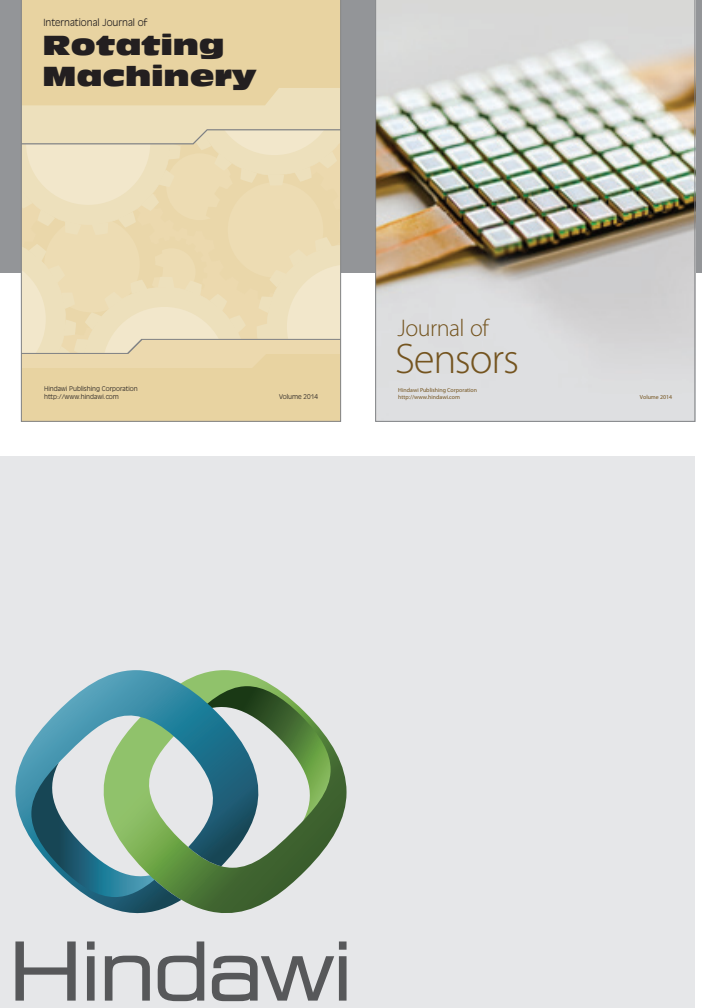

Submit your manuscripts at http://www.hindawi.com
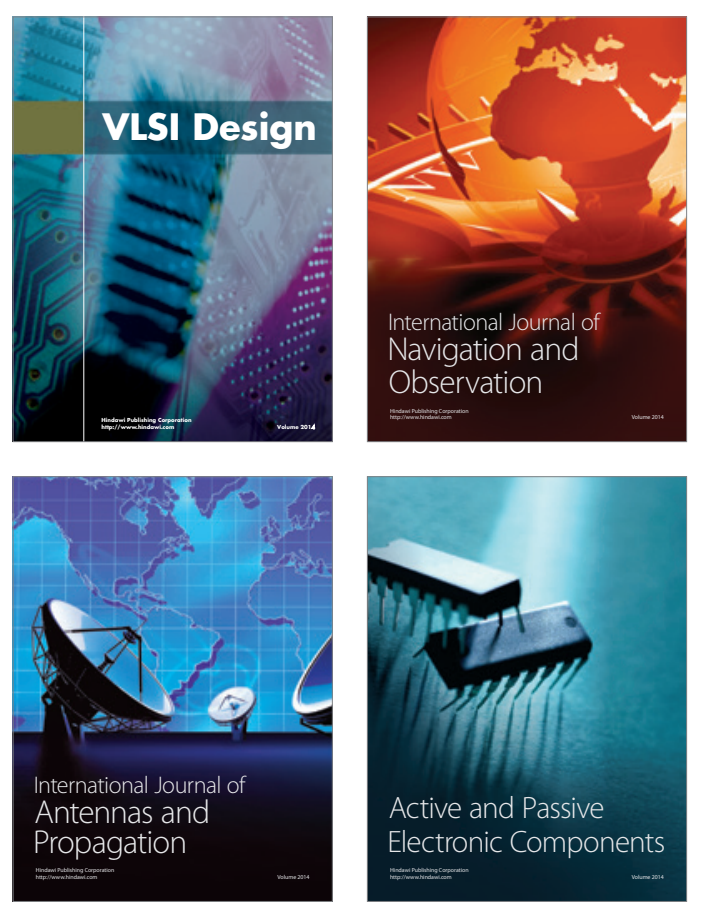
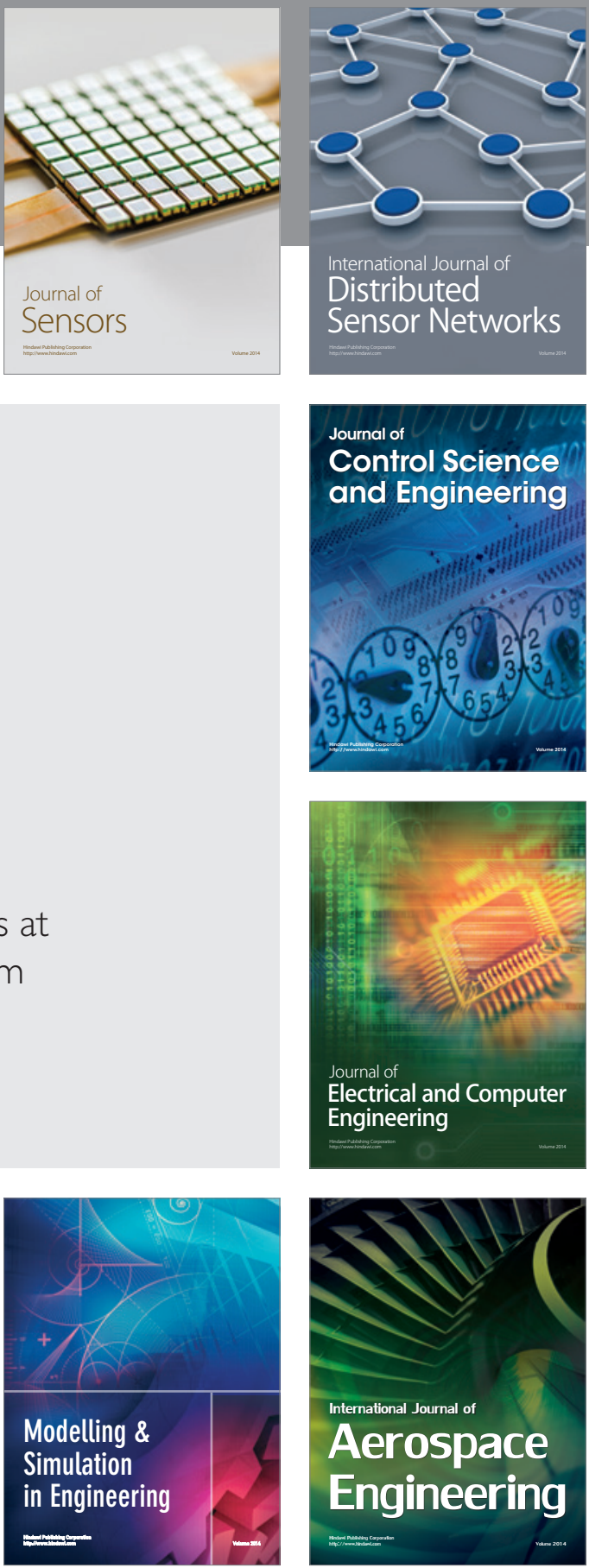

Journal of

Control Science

and Engineering
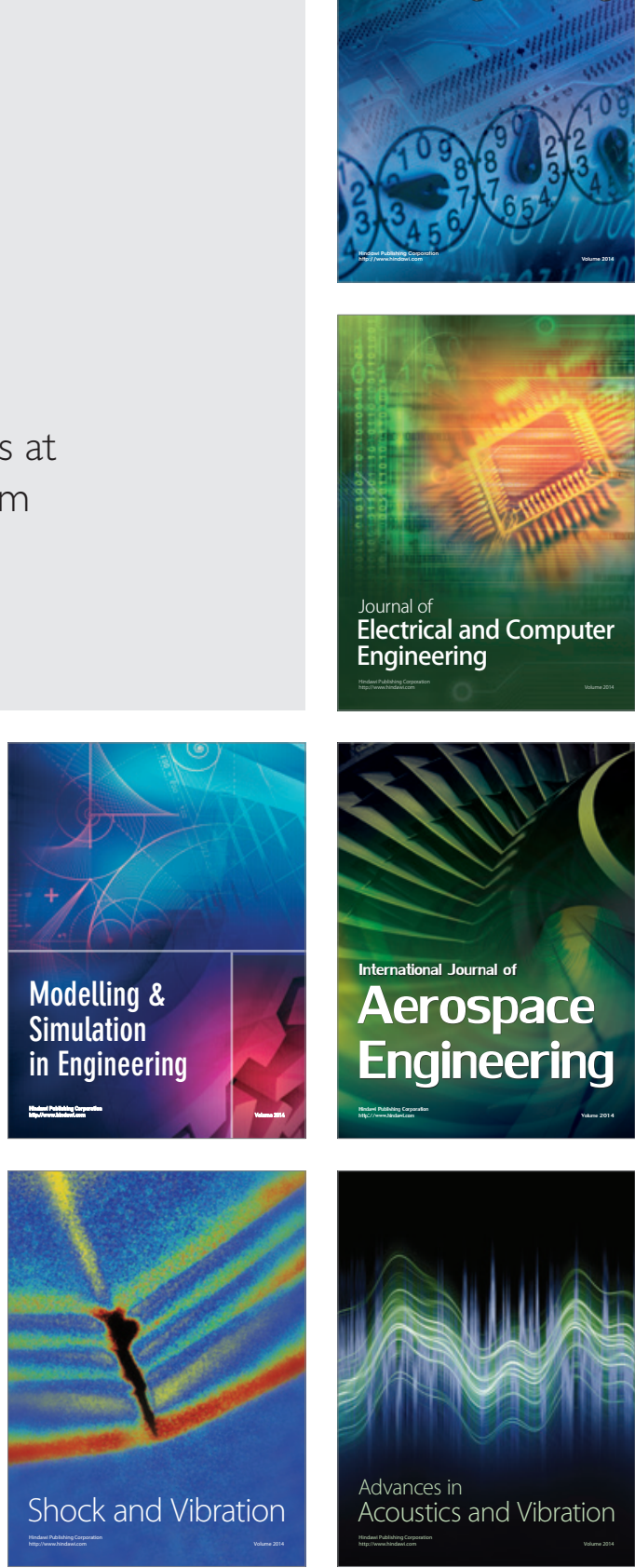